\title{
Recurrent pulmonary synovial sarcoma effectively treated with amrubicin: A case report
}

\author{
HIROAKI SATOH ${ }^{1}$, NORIO TAKAYASHIKI ${ }^{2}$, TOSHIHIRO SHIOZAWA ${ }^{3}$, KUNIHIKO MIYAZAKI ${ }^{3}$, \\ GEN OHARA $^{1}$, KATSUNORI KAGOHASHI ${ }^{1}$, KOICHI KURISHIMA ${ }^{1}$, SHINTARO SUGITA $^{4}$, \\ TOMOYUKI AOYAMA ${ }^{4}$, TADASHI HASEGAWA ${ }^{4}$ and NOBUYUKI HIZAWA ${ }^{3}$
}

\author{
Divisions of ${ }^{1}$ Respiratory Medicine and ${ }^{2}$ Pathology, Mito Medical Center, University of Tsukuba, Mito, Ibaraki 210-0015; \\ ${ }^{3}$ Division of Respiratory Medicine, Faculty of Medicine, University of Tsukuba, Tsukuba, Ibaraki 305-8575; \\ ${ }^{4}$ Department of Pathology, Sapporo Medical University, Sapporo, Hokkaido 060-8556, Japan
}

Received May 29, 2014; Accepted January 29, 2015

DOI: $10.3892 /$ etm.2015.2308

\begin{abstract}
Pulmonary synovial sarcoma is a rare but aggressive disease. The present study describes the case of a 68-year-old female with pulmonary synovial sarcoma. The patient was mistakenly treated for small cell lung cancer due to false-positive staining for synaptophysin and cluster of differentiation 56. Despite severe myelotoxicity, platinum-containing chemotherapies (cisplatin plus irinotecan and carboplatin plus etoposide) were not effective. As a third-line therapy, the patient received amrubicin (AMR) monotherapy. A partial response was achieved, and the patient was able to undertake ordinary daily life at home for 13 months from the initiation of AMR chemotherapy. Due to the atypical clinical condition and unusual response to chemotherapy in this patient, the pathological examination was repeated. The SS18 split-signal was detected in fluorescence in situ hybridization analysis. From these results, the tumor was diagnosed as a monophasic synovial sarcoma. To the best of our knowledge, this is the first case of a patient with pulmonary synovial sarcoma who underwent successful treatment with AMR. The present case could demonstrate a specific chemosensitivity of such a rare tumor.
\end{abstract}

\section{Introduction}

Although rare, primary synovial sarcoma is one of the most common variants of pulmonary sarcoma. Primary synovial sarcoma usually derive from soft tissue, most commonly in young individuals (1). Diagnosis requires a tissue sample in the form of a needle or open biopsy. Surgical resection is the standard primary treatment, and a 5-year distant metastasis-free

Correspondence to: Professor Hiroaki Satoh, Division of Respiratory Medicine, Mito Medical Center, University of Tsukuba, Miya-machi 3-2-7, Mito, Ibaraki 210-0015, Japan

E-mail: hirosato@md.tsukuba.ac.jp

Key words: pulmonary synovial sarcoma, chemotherapy, amrubicin survival of $>60 \%$ may be achievable $(2,3)$. However, recurrence remains a major problem and is responsible for treatment failures (4). Clinically, pulmonary synovial sarcoma may be characterized as an aggressive tumor; to the best of our knowledge, there has not been a single report of a long-term follow-up of a patient with pulmonary synovial sarcoma. The present study describes the case of a patient with recurrent pulmonary synovial sarcoma, who was effectively treated with amrubicin (AMR) monotherapy as a third-line chemotherapy following cisplatin and irinotecan and carboplatin and etoposide.

\section{Case report}

A 68-year-old female was admitted to hospital with a cough, dyspnea on exertion and a large, right-sided pleural effusion. The patient was a non-smoking housewife with no history of asbestos exposure. Thirty-five months prior to this admission, the patient underwent a left upper lobectomy and mediastinal lymph node dissection for a mass in the left upper lobe of the lung. The pathological diagnosis of the resected tumor was small cell lung cancer (SCLC) due to its weakly positive staining for synaptophysin and cluster of differentiation (CD) 56. Following the surgery, the patient received three courses of chemotherapy with cisplatin and irinotecan; however, local recurrence developed at the left mediastinum. The patient was therefore referred to the Mito Medical Center (Mito, Japan) to receive further chemotherapy. Chest computed tomography (CT) revealed a large mass in the left mediastinum; the mediastinum was shifted to the right and large, left-sided pericardial and pleural effusions were observed (Fig. 1). The patient then received two courses of chemotherapy with carboplatin and etoposide, but the response was evaluated as a progressive disease, despite severe myelosuppression (grade 4 thrombocytopenia in the National Cancer Institute-Common Toxicity Criteria), which required platelet transfusion. The patient additionally developed cardiac tamponade, which was successfully treated with tube drainage. Informed consent was obtained from the patient's family.

As a third-line chemotherapy, to avoid severe myelosuppression, weekly AMR monotherapy was selected: Administration of $35 \mathrm{mg} / \mathrm{m}^{2}$ AMR three times during a four-week period (5). 

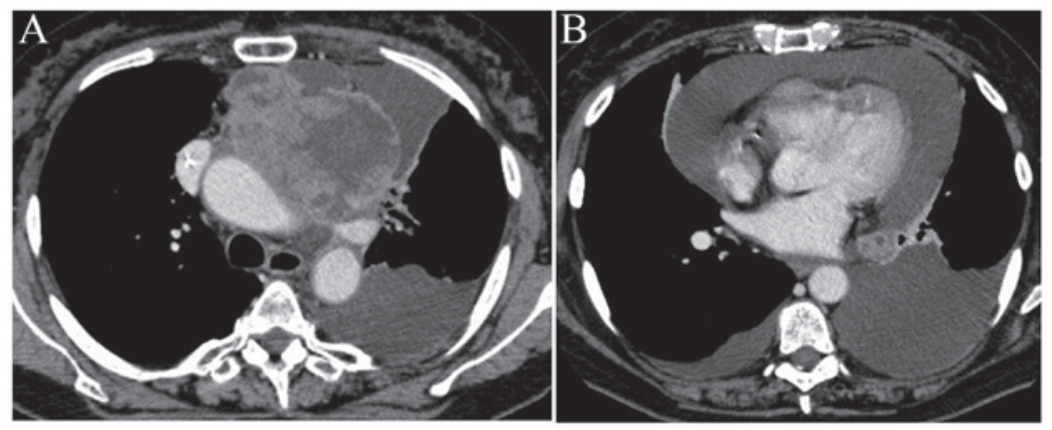

Figure 1. Chest computed tomography revealed (A) a large mass in the left mediastinum, with the mediastinum shifted to the right; and (B) pericardial and pleural effusion.

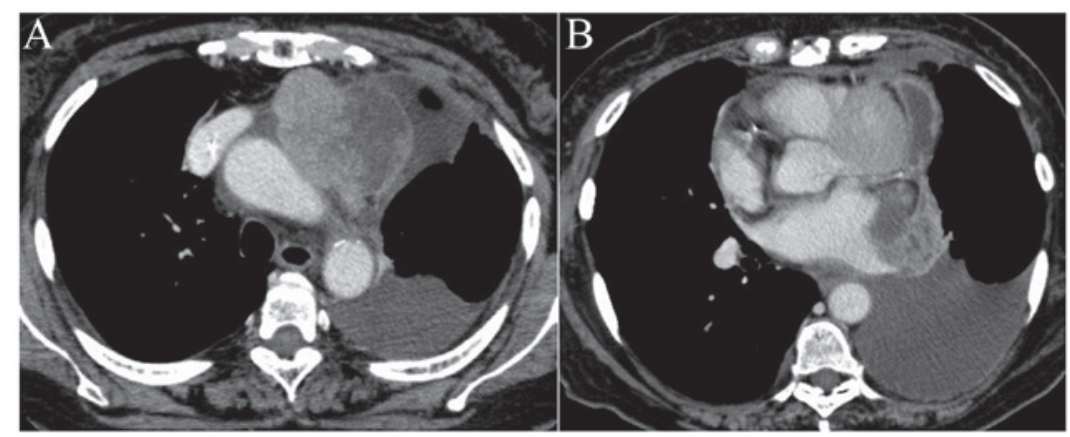

Figure 2. Chest computed tomography following two courses of chemotherapy showed (A) a partial response and (B) disappearance of the pericardial fluid.

Myelotoxicity could then be evaluated prior to the next AMR administration. A partial response was achieved (Fig. 2) with no severe myelotoxicity. The patient received eight courses of the chemotherapy and was able to undertake ordinary daily life at home for 13 months from the initiation of the AMR chemotherapy. Local recurrence at the left mediastinum then developed and the patient succumbed of the disease, 50 months after the initial surgical resection of the tumor.

Due to the atypical clinical condition and unusual response to chemotherapy in this patient, the pathological examination was repeated. The resected tumor was composed of spindle cells with increased nuclear chromatin arranged uniformly (Fig. 3). Immunostaining with synaptophysin and CD56 revealed negative results. The tumor cells were positive for vimentin and B-cell lymphoma 2, but negative for cytokeratin AE1/AE3, $\alpha$-smooth muscle actin, desmin, S-100, CD34, chromogranin A and synaptophysin. The SS18 split-signal was detected in fluorescence in situ hybridization analysis. From these results, the tumor was diagnosed as a monophasic synovial sarcoma.

\section{Discussion}

Pulmonary synovial sarcoma is a rare, aggressive primary tumor. The neoplasm was first described in 1995 by Zeren et al (6) as a distinctive pulmonary tumor of the lung, sharing histological and immunohistochemical characteristics with the monophasic synovial sarcoma of the soft tissue. Chest pain, dyspnea, cough and hemoptysis are the most common symptoms in patients with pulmonary synovial sarcoma (7); however, certain patients do not present with any
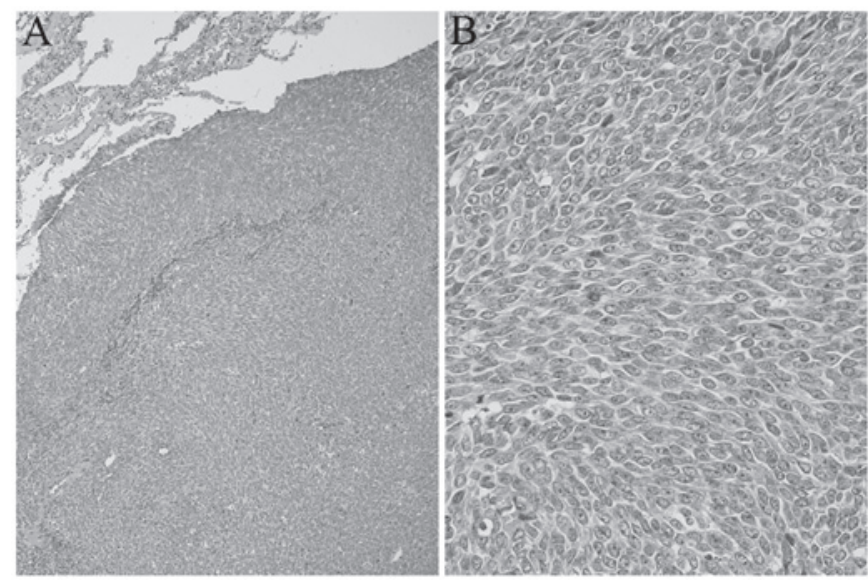

Figure 3. Pathological examination using the resected tumor, which was composed of spindle cells with increased nuclear chromatin arranged uniformly: (A) Low-power field (x100) and (B) high-power field (x200), stained with hematoxylin-eosin.

symptoms (8). Radiologically, pulmonary synovial sarcoma appears mostly as well-circumscribed nodules of considerable size (9). In order to diagnose pulmonary synovial sarcoma, it is mandatory to exclude extrathoracic primary synovial sarcoma by whole-body survey. Detection of the $t(X ; 18)$ chromosomal translocation can confirm the diagnosis $(10,11)$. In the patient in the present case, no primary site other than the lung was found and positive split-signals from an SS18 probe were detected; a diagnosis of synovial sarcoma was thus confirmed. The patient was misdiagnosed with SCLC due to weakly positive staining for synaptophysin and CD56. SCLC is a tumor arising in a 
relatively large bronchus and predominantly occurs in patients with a smoking habit. In Japan, the majority of patients with SCLC have been males with a history of smoking (12). The present case was a female patient without a smoking habit. In addition, the patient had no history of exposure to carcinogenic chemicals such as asbestos. The poor response to platinum-containing chemotherapy was also unusual. The SCLC in this patient was therefore somewhat different from that usually observed. The histopathology of the resected tumor was re-evaluated and a correct diagnosis was achieved.

Surgical removal of the resectable tumor is the choice of treatment for pulmonary synovial sarcoma, and the prognosis of the disease is poor if the complete surgical resection cannot be performed. Bacha et al (13) reported that successful complete resection was significantly associated with increased survival, and that the size and grade of the tumors were not. As there have been no randomized control trials, the role of chemotherapy for unresectable and recurrent tumors remains unclear.

AMR is an anthracycline derivative with a structure similar to that of doxorubicine (14). Anthracyclines are widely used in the treatment of a variety of sarcomas (15). Although the cumulative dose-limiting cardiotoxicity is a major obstacle to its use (16), AMR has shown considerably less cardiotoxicity than doxorubicin in experimental models $(17,18)$ and clinical trials $(19,20)$. AMR has been demonstrated to be effective for previously treated SCLC $(21,22)$. In the clinical course of the present case, the patient developed myelotoxicity, which required a platelet transfusion subsequent to the second course of the second-line chemotherapy with carboplatin and etoposide. AMR was therefore administered weekly (three times in four weeks at $35 \mathrm{mg} / \mathrm{m}^{2}$ ), taking the severe myelotoxicity of the second-line chemotherapy into consideration.

To the best of our knowledge, this is the first case of a patient with pulmonary synovial sarcoma to be effectively treated with AMR. Following AMR administration, the tumor exhibited a decrease in size on serial chest CT scan, and the survival benefit was obtained without any severe myelotoxicity and cardiotoxicity, although the patient had a history of cardiac tamponade. The present case could demonstrate a specific chemosensitivity of such a rare tumor.

\section{References}

1. Siegel HJ, Sessions W, Casillas MA Jr, et al: Synovial sarcoma: clinicopathologic features, treatment, and prognosis. Orthopedics 30: 1020-1025, 2007.

2. Liu CY, Yen CC, Chen WM, et al: Soft tissue sarcoma of extremities: the prognostic significance of adequate surgical margins in primary operation and reoperation after recurrence. Ann Surg Oncol 17: 2102-2111, 2010.

3. Stojadinovic A, Leung DH, Hoos A, Jaques DP, Lewis JJ and Brennan MF: Analysis of the prognostic significance of microscopic margins in 2,084 localized primary adult soft tissue sarcomas. Ann Surg 235: 424-434, 2002.
4. Morrison BA: Soft tissue sarcomas of the extremities. Proc (Bayl Univ Med Cent) 16: 285-290, 2003.

5. Kitagawa C, Saka H, Kajikawa S, Mori K, Oki M and Suzuki R: Phase I and pharmacologic study of weekly amrubicin in patients with refractory or relapsed lung cancer: Central Japan Lung Study Group (CJLSG) 0601 trial. Cancer Chemother Pharmacol 69: 1379-1385, 2012.

6. Zeren H, Moran CA, Suster S, Fishback NF and Koss MN: Primary pulmonary sarcomas with features of monophasic synovial sarcoma: A clinicopathological, immunohistochemical and ultrastructural study of 25 cases. Hum Pathol 26: 474-480, 1995.

7. Hartel PH, Fanburg-Smith JC, Frazier AA, et al: Primary pulmonary and mediastinal synovial sarcoma: A clinicopathologic study of 60 cases and comparison with five prior series. Mod Pathol 20: 760-769, 2007.

8. Watzka SB, Setinek U, Prosch H and Müller MR: Primary synovial sarcoma of the lung as an incidental finding. Interact Cardiovasc Thorac Surg 9: 1026-1028, 2009.

9. Frazier AA, Franks TJ, Pugatch RD and Galvin JR: From the archives of the AFIP: Pleuropulmonary synovial sarcoma. Radiographics 26: 923-940, 2006.

10. dos Santos NR, de Bruijn DR and van Kessel AG: Molecular mechanisms underlying human synovial sarcoma development. Genes Chromosomes Cancer 30: 1-14, 2001.

11. Miura Y, Keira Y, Ogino J, et al: Detection of specific genetic abnormalities by fluorescence in situ hybridization in soft tissue tumors. Pathol Int 62: 16-27, 2012.

12. Cuffe S, Moua T, Summerfield R, Roberts H, Jett J and Shepherd FA. Characteristics and outcomes of small cell lung cancer patients diagnosed during two lung cancer computed tomographic screening programs in heavy smokers. J Thorac Oncol 6: 818-822, 2011.

13. Bacha EA, Wright CD, Grillo HC, et al: Surgical treatment of primary pulmonary sarcomas. Eur J Cardiothorac Surg 15: 456-460, 1999.

14. Shah CM: Amrubicin: A synthetic anthracyclin analogue in the treatment of extensive stage small cell lung cancer. Recent Pat Anticancer Drug Discov 4: 241-245, 2009.

15. Walczak BE and Irwin RB: Sarcoma chemotherapy. J Am Acad Orthop Surg 21: 480-491, 2013.

16. Weiss RB: The anthracyclines: Will we ever find a better doxorubicin? Semin Oncol 19: 670-686, 1992.

17. Suzuki T, Minamide S, Iwasaki T, Yamamoto $\mathrm{H}$ and Kanda $\mathrm{H}$ : Cardiotoxicity of a new anthracycline derivative (SM-5887) following intravenous administration to rabbits: Comparative study with doxorubicin. Invest New Drugs 15: 219-225, 1997.

18. Noda T, Watanabe T, Kohda A, Hosokawa S and Suzuki T: Chronic effects of a novel synthetic anthracycline derivative (SM-5887) on normal heart and doxorubicin-induced cardiomyopathy in beagle dogs. Invest New Drugs 16: 121-128, 1998.

19. Fulbright JM, Huh W, Anderson P and Chandra J: Can anthracycline therapy for pediatric malignancies be less cardiotoxic? Curr Oncol Rep 12: 411-419, 2010.

20. Ogawara D, Fukuda M, Nakamura Y and Kohno S: Efficacy and safety of amrubicin hydrochloride for treatment of relapsed small cell lung cancer. Cancer Manag Res 2: 191-195, 2010.

21. Onoda S, Masuda N, Seto T, et al; Thoracic Oncology Research Group Study 0301: Phase II trial of amrubicin for treatment of refractory or relapsed small-cell lung cancer: Thoracic Oncology Research Group Study 0301. J Clin Oncol 24: 5448-5453, 2006.

22. Harada T, Oizumi S, Ito K, et al; Hokkaido Lung Cancer Clinical Study Group: A phase II study of amrubicin as a third-line or fourth-line chemotherapy for patients with non-small cell lung cancer: Hokkaido Lung Cancer Clinical Study Group Trial (HOT) 0901. Oncologist 18: 439-445, 2013. 